\title{
DISTRIBUTION AND MOTIONS OF ATOMIC HYDROGEN IN LENTICULAR
} GALAXIES

\author{
Hugo van Woerden, Wim van Driel and U1rich J. Schwarz \\ Kapteyn Astronomical Institute \\ Groningen University \\ Groningen, the Netherlands
}

ABSTRACT

We report thr results of HI observations of eleven gas-rich SO/SOa galaxies with the Westerbork Synthesis Telescope. The majority of these galaxies have most of their hydrogen outside the optical body, in annular configurations with diameters $\sim 2$ times the optical. These outer gas rings are often clumped, incomplete, and in approximately circular motion. They may represent the remnants of primordial, often warped, gas disks; or they may have formed from gas (or dwarf galaxies) accreted recently. Optical spectra could discriminate between these possibilities.

A few objects have filled gas disks, and several have inner HI rings with radii $\sim 0.4 \times$ optical. Two objects have peculiar distributions suggesting, respectively, tidal effects and stripping. However, stripping by intergalactic gas appears not to be a major current process.

\section{INTRODUCTION}

The gas content of lenticular (or, equivalently, so) galaxies plays a key role in the problem of their origin and evolution.

Faber and Gallagher (1976) estimate that a galaxy of blue luminosity $L_{B}$ should, through mass loss from evolving stars, in a Hubble time produce an amount of interstellar gas, $M_{g}$, given by $M_{g} / M_{\odot} \sim 0.1 L_{B} / L_{\odot}$. Such amounts should be readily detectable, but are rarely observed in So galaxies. Faber and Gallagher consider several ways to remove the gas. Star formation would probably make the SO's bluer than they are observed to be. Stripping by collisions with other galaxies or with intergalactic gas might work in clusters, but not for field so's. Faber and Gallagher conclude that hot, supernova-driven galactic winds are probably responsible for the lack of gas in $\mathrm{SO}^{\prime} \mathrm{s}$.

Larson, Tinsley and Caldwell (1980) calculate that star formation will exhaust the disk gas in most spiral galaxies in a fraction of the Hubble time. They suggest that spirals are replenished from gas-rich envelopes consisting of tidal debris, dwarf companions and/or remnants of primordial gas; and that SO's were formed from disk galaxies which early lost their envelopes and then consumed their gas by star formation. Larson et al. consider that gas produced later by evolving stars may be removed by intergalactic gas, by galactic winds, or by short bursts of star formation. 
Thus, the absence of gas traditionally assumed in so galaxies may be theoretically understood. But what is the observational situation?

Since 1975, major surveys of the HI content of SO and SO/a galaxies have been carried out at Arecibo, Green Bank and Parkes. Some 200 objects have been observed, and about 40-50 of these detected. The $\mathrm{M}_{\mathrm{HI}} / \mathrm{L}_{\mathrm{B}}$ values measured range between $<0.001$ and 0.6 . Clearly, the gas content of $S O$ and So/a galaxies varies over a wide range. This fact suggests that the HI component in these galaxies may be a transient phenomenon, and that we see them in different stages of evolution.

In an attempt to understand the origin and evolution of the gas in lenticular galaxies, we have measured the HI distribution and velocity field in a large number of such galaxies with the Westerbork Synthesis Radio Telescope. The present paper summarizes the current results of this program, and draws some tentative conclusions.

\section{WESTERBORK OBSERVATIONS}

For our Westerbork program, we selected galaxies of morphological type SO or So/a (as listed in the Second Reference Catalogue, RC2), having a good HI detection in the recent surveys, positive declination, and sufficient angular size. Our sample spans considerable ranges in $\mathrm{M}_{\mathrm{HI}} / \mathrm{L}_{\mathrm{B}}$ and in luminosity. Table 1 lists 11 objects for which maps are now available; it includes a few objects observed by colleagues at Groningen, who kindly made their results available for the present discussion. Several more objects have been observed, and the program continues. The Revised Shapley Ames (RSA) types listed show that most of our objects are genuine $\mathrm{SO}^{\prime} \mathrm{s}$ or $\mathrm{SO} / \mathrm{a}^{\prime} \mathrm{s}$.

The observations have $40 \mathrm{~km} / \mathrm{s}$ velocity resolution; the angular resolution is $0 ! 4$ in $\alpha$ and $0 ! 4 / \sin \delta$ in $\delta$ for most objects.

\section{THE HYDROGEN DISTRIBUTIONS AND MOTIONS}

In this section, we first describe the results obtained in two typical cases: NGC 4203 and 4262. Next we summarize and discuss the gas distributions and motions found in our sample of 11 galaxies.

\subsection{NGC 4203 and 4262}

NGC 4203 is a pure So galaxy of optical diameter $D_{25}=3: 6$ and inclination $1 \sim 30^{\circ}$. The hydrogen in this galaxy has been mapped at Arecibo with $3^{\prime}$ resolution by Burstein and Krumm (1981). We find (Fig. 1) most of the gas to lie outside the optical body, in an annular configuration of $6: 5$ diameter, $1 . e$. almost twice the optical. This outer HI ring is incomplete and strongly clumped. If its true shape is roughly circular, it has an inclination of $-60^{\circ}$ to the plane of the sky, and of $30^{\circ}$ or $90^{\circ}$ to the stellar disk. We also observe an inner ring, of dia- 
Figure 1. Hydrogen distributions (left) and velocity fields (right) for two galaxies. Column density contour values are $1.0,2.0,3.0 \times$ $10^{20}$ atoms $/ \mathrm{cm}^{2}$ for NGC 4203; and $1.4,2.8, \ldots 7.0 \times 10^{20}$ atoms $/ \mathrm{cm}^{2}$ for NGC 4262 . Velocities (in km/s) are heliocentric. The dashed ellipses are $25 \mathrm{mag} / \operatorname{arcsec}^{2}$ isophotes from RC2; the shaded ellipses show the angular resolution of the HI observations. Linear scales are for distances based on $\mathrm{H}_{\mathrm{O}}=100 \mathrm{~km} \mathrm{~s}^{-1} \mathrm{Mpc}^{-1}$.

The HI distributions are dominated by incomplete, clumpy, tilted outer-ring configurations. The velocity fields indicate that these rings are in approximately circular rotation.

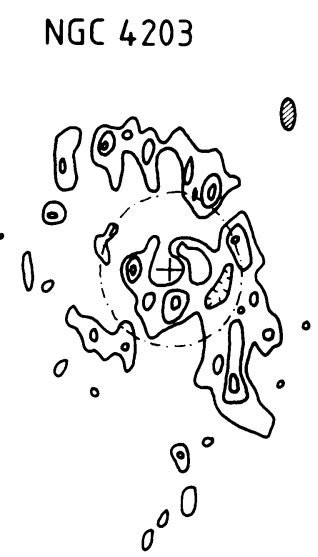

NGC 4262

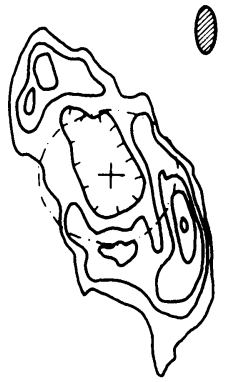

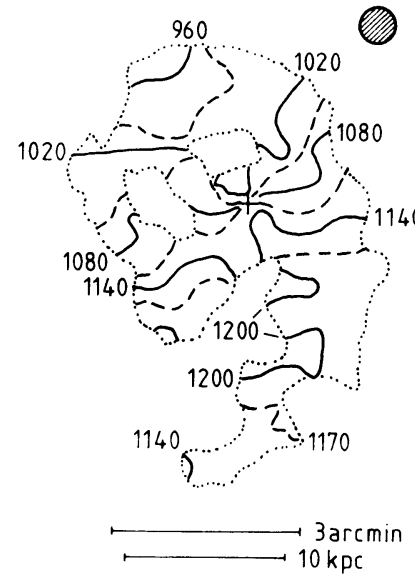

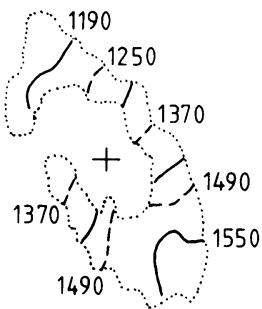

TABLE 1

Structural and dynamical properties of HI in lenticular galaxies

\begin{tabular}{|c|c|c|c|c|c|c|c|c|c|c|c|}
\hline $\begin{array}{l}\text { (1) } \\
\text { Name } \\
\text { NGC }\end{array}$ & $\begin{array}{l}\text { (2) } \\
\text { Morphol. } \\
(\mathrm{RC2})\end{array}$ & $\begin{array}{l}\text { type } \\
\text { (RSA) }\end{array}$ & $\begin{array}{l}\text { Features in } \\
c r, D_{r}^{\prime}, x\end{array}$ & $\begin{array}{l}\text { (3) } \\
\text { HI } \\
\text { (d) }\end{array}$ & $\begin{array}{l}\text { distribution } \\
R, D_{R}^{\prime}, \chi^{T}\end{array}$ & $\begin{array}{l}(4) \\
\text { Notes }\end{array}$ & $\begin{array}{l}(5) \\
h R_{R} \\
k p c\end{array}$ & $\begin{array}{l}(6) \\
v_{\mathrm{rm}}{ }_{\mathrm{r}}^{\mathrm{t}}\end{array}$ & $\begin{array}{l}(7) \\
\mathrm{hP}^{\mathrm{R}} \\
10^{9} \mathrm{yr}\end{array}$ & $\begin{array}{c}(8) \\
\mathrm{hM}_{\mathrm{R}} \\
10 \mathrm{PM}_{\odot}\end{array}$ & $\begin{array}{c}(9) \\
M_{R} / h^{2} L_{B} \\
M_{\odot} / L_{\odot}\end{array}$ \\
\hline $\begin{array}{l}1023 \\
2273 \\
2787 \\
3900 \\
3941 \\
3998\end{array}$ & $\begin{array}{l}\text { S B } 0- \\
\text { SABrs0: } \\
\text { S Br O+ } \\
\text { SA r O+ } \\
\text { S Bs O+ } \\
\text { SA r O? }\end{array}$ & $\begin{array}{l}\text { SBO } 1 \\
\text { SBOa } \\
\text { S a(r) } \\
\text { SBOal-2 } \\
\text { S.0 1 }\end{array}$ & $\begin{array}{l}r_{0}, 0.4,< \\
r, 0.4,< \\
r ?\end{array}$ & $\begin{array}{l}d \\
d\end{array}$ & $\begin{array}{l}\mathrm{R}, 2.5,<\mathrm{T} \\
\mathrm{R}, 0.8,< \\
\mathrm{R}, 2.5:, 60^{\circ} \\
\mathrm{R}, 1.6,< \\
\mathrm{R} 0.7:, 70^{\circ}\end{array}$ & 2 vel. systems & $\begin{array}{r}11 \\
8 \\
4:\end{array}$ & $\begin{array}{l}220 \\
160 \\
280\end{array}$ & $\begin{array}{l}1.1 \\
0.2 \\
\\
0.3 \\
0.3 \\
0.1\end{array}$ & $\begin{array}{r}110 \\
80 \\
\\
120 \\
50 \\
70:\end{array}$ & $\begin{array}{c}14 \\
9 \\
12:\end{array}$ \\
\hline $\begin{array}{l}4203 \\
4262 \\
4694 \\
5084 \\
7013\end{array}$ & 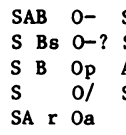 & 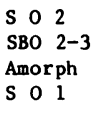 & $\begin{array}{l}\quad \mathrm{r}, 0.4,< \\
\mathrm{c} \\
\mathrm{r}_{0}, 0.4,25^{\circ}:\end{array}$ & d & $\begin{array}{l}\mathrm{R}, 1.8,30^{\circ} \\
\mathrm{R}, 1.7,40^{\circ} \\
\mathrm{R}, 2.5,<\mathrm{T} \\
\mathrm{R}, 0.85,<\end{array}$ & $\begin{array}{c}\text { V1rgo Cluster } \\
\text { Virgo Cluster } \\
\qquad \star \star \star\end{array}$ & $\begin{array}{r}11 \\
7\end{array}$ & $\begin{array}{l}150 \\
240\end{array}$ & $\begin{array}{l}0.4 \\
0.2 \\
\\
0.5 \\
0.2\end{array}$ & $\begin{array}{r}60 \\
90 \\
600 \\
40\end{array}$ & $\begin{array}{r}10 \\
27 \\
60 \\
7\end{array}$ \\
\hline
\end{tabular}

Key to Table 1

(2) Morphological types, from Second Reference Catalogue (RC2) and Revised Shapley Ames Catalog (RSA)

(3) Features in HI distribution: $c=$ concentration at centre; $r=1$ inner ring; $r=r$ coinciding with optical inner ring; $d,(d)=f i l l e d$, resp. partly filled gas disk; $R=$ outer ring; $T=$ tall. $D_{r}^{\prime}$, $D_{k}$ give ring diameters relative to $D_{25}$ (from $\mathrm{RC} 2$ ); $\chi$ = inclination of ring (assumed circular) relative to optical disk; < means $\chi$ is small.

(4) * NGC 3900: Data In columns (5)-(9) given for edge of HI disk.

$\star \star$ NGC 3998: Diameter and category of HI ring uncertain.

$\star \star \star$ NGC 7013: Inner ring tilted or motions non-circular.

(5)-(7) Linear radius $R_{R}$, rotation speeed $V_{\text {, }}$, and period $P_{R}$ of outer HI ring.

(8), (9) Total mass $M_{R}$ within outer ring (assuming spherical distribution), and ratio to blue luminosity $L_{B}$. 
meter $0.4 \mathrm{D}_{25}$ and negligible tilt relative to the disk. A smoothed map shows weak disk emission between both rings. The velocity field is somewhat irregular, but circular motions in a warped disk with a flat rotation curve give a reasonable fit.

NGC 4262 is an almost face-on $\left(i<30^{\circ}\right)$, barred so galaxy of $2: 2$ diameter, $3^{\circ} \mathrm{NW}$ of the centre of the Virgo Cluster. Its hydrogen (Fig. 1) lies in an outer ring of $3: 7$ diameter (i.e. $1.7 \times$ optical) and $60^{\circ}$ inclination (if circular), hence tilted $>30^{\circ}$ relative to the disk. The ring is fairly complete, but quite uneven in brightness. The velocity field bears the signature of solid-body rotation, indicating a circular ring in circular motion.

\subsection{A summary for 11 galaxies}

Table 1 summarizes our results for 11 galaxies. Among these, 8 have most of their hydrogen in outer-ring configurations ( $R$ ), with diameters between 0.8 and 2.5 times the optical $\left(\mathrm{D}_{25}\right)$. In general, these rings are roughly concentric with the optical disk; however, if circular in shape, several are strongly inclined to the disk. The density distributions in these rings are quite uneven; some are strongly clumped, and of ten parts of the ring are not detected. The velocities measured in the rings closely follow the pattern of circular rotation; in NGC 1023 two velocity systems are observed. In section 4 we discuss the rotation speeds and periods, and the galaxy masses derived.

Four galaxies have inner HI rings ( $r$ ) of diameters about 0.4 times the optical disk. Except in NGC 3900, these inner rings combine with outer HI rings. In NGC 3900 and 7013, they approximately coincide with optical (probably stellar) inner-ring features. In NGC 7013 the velocity field indicates that the inner HI ring is tilted relative to the disk, or that it possesses non-circular motions.

HI distribution and motions in NGC 3998 indicate a ring observed edge-on and highly inclined to the optical body (cf. Knapp, this symposium). The ring diameter is uncertain because of the edge-on situation.

In four galaxies we observe a disk of hydrogen, in addition to an inner and/or outer ring. In NGC $420 \overline{3}$ we find a faint, extended disk. The HI disks in NGC 2273, 3900 and 7013 are about as large as the optical disks; these 3 galaxies, however, may be early-type spirals.

Two galaxies in Table 1 have exceptional HI distributions. NGC 1023 (Sancisi et al., in preparation) shows an incomplete outer ring in circular motion, an anomalous velocity system, and a bright HI tail. The tail and the anomalous-velocity system may be due to tidal action of NGC 1023 on a nearby, smaller late-type galaxy. NGC 4694 has a narrow, clumpy HI tall and an unresolved central condensation of gas. This unique distribution and the galaxy's location in the Virgo Cluster ( $5^{\circ}$ $\mathrm{SE}$ of the centre) suggest stripping by intergalactic ram pressure as a possible cause. 
4. ROTATION SPEEDS AND PERIODS; MASSES AND M/L RATIOS

The observed outer gas rings allow us to derive some dynamical properties of gas-rich lenticular galaxies. The results (Table 1) are given for distances based on a Hubble constant $\mathrm{H}_{\mathrm{O}}=100 \mathrm{~h} \mathrm{~km} \mathrm{~s} \mathrm{spc}^{-1}$.

The outer HI rings have radii $R_{R}$ ranging from 6 to $27 \mathrm{~h}^{-1} \mathrm{kpc}$. Assuming circular ring shapes to derive inclinations, we find rotation velocities $V_{\text {rot }}$ between $\sim 150$ and $\sim 300 \mathrm{~km} / \mathrm{s}$. The present sample shows no
Tully-Fisher relation between rotation velocity and luminosity, a finding similar to that of Dressler and Sandage (1982, and this symposium) from optical rotation curves for another sample of so's. The rotation periods of the outer HI rings range between 2 and $10 \mathrm{~h}^{-1} 10^{8}$ years.

Assuming spherical mass distributions, we derive total masses inside the outer $H I$ rings, $M_{R}=V_{\text {fot }}^{2} R_{R} / G$, ranging from 0.4 to $6 \mathrm{~h}^{-1} 10^{11}$
solar masses. The total-mass-to-blue-light ratios cluster around $10 \mathrm{~h}$; two values are much higher. We find no correlation between $M_{R} / L_{B}$ and relative ring diameter, $D_{R} / D_{25^{\circ}}$

\section{ORIGIN AND EVOLUTION OF THE GASEOUS COMPONENT}

In this section we draw some tentative conclusions from the observational results summarized in section 3 and Table 1 .

1) Our major finding is that in many gas-rich lenticulars most of the hydrogen is located in the outer parts, or even outside, of the luminous body. This strongly suggests that stripping by intergalactic ram pressure is not a major current process in so galaxies. For such stripping should primarily remove the gas in the outer, rather than the inner, parts. The only likely stripper in our sample is NGC 4694 , a galaxy in the Virgo Cluster. However, the other Virgo member, NGC 4262 , has all of its gas in a well-formed outer ring structure!

2) The frequent outer rings might well be interpreted as a stage in the process of gas removal by galactic winds. However, it is unclear that such winds would produce the observed uneven distributions in the outer HI rings. Also, the circular motions observed may be inconsistent with angular momentum conservation in an outward gas flow (although this argument may be invalid in non-axisymmetric cases).

3) Another possibility to be considered is that of accretion of intergalactic gas clouds, tidal debris or dwarf companions (cf. Silk and Norman, 1979) by a formerly gas-poor lenticular galaxy. The uneven structure of the outer rings may suggest dwarf capture. The close-tocircular motions suggest that capture occurred a few periods, that is: about $10^{9}$ years, ago. The inner HI rings observed in a few cases might be due to earlier accretion, cf. the Spindle galaxy, NGC 2685 (Shane, 1980). 
4) Finally the outer rings may represent the remnants of primordial, often warped gas disks, of which the dense inner parts have been used up in star formation, while the tenuous outer parts never gave birth to stars. (For removal of the gas produced later in the inner parts by stellar evolution, a galactic wind may then still be required.) This scenario is partly similar to that of Larson, Tinsley and Caldwell (1980) summarized in section 1. However, most of our gas-rich SO's apparently do have gas-rich envelopes, which somehow may have failed to replenish the inner disks.

These are tentative suggestions, which will have to be analyzed in detail before they can lead to firm conclusions. However, an important observational test appears evident. Comparison of the sense of rotation in the outer gas rings with that in the inner, stellar disks should decide for or against scenario 3). Opposite senses of rotation would require accretion; if the senses always agree, recent accretion of dwarfs or intergalactic gas can probably be ruled out.

Another item for further study is what the gas distributions tell us about the place of so galaxies in the morphological sequences. For this purpose, we have undertaken a companion program on Sa galaxies, as part of a comparison between lenticulars and spirals. Also, the relationship of the outer HI rings to the phenomenon of optical outer rings requires investigation.

\section{ACKNOWLEDGEMENTS}

First, we thank Jill Knapp for her vital contributions to this program. She has freely provided us with unpublished detections, and shared in many of our analyses and discussions. We further thank Renzo Sancisi and Seth Shostak for making their results on NGC 1023 and 2787 available, and for much help and discussion. Jay Gallagher and Nathan Krumm collaborated on individual galaxies. Beatrice Tinsley, Bob Sanders, James Binney and Scott Tremaine contributed through discussions.

Wim van Driel is supported by ZWO through the ASTRON foundation. Jill Knapp's visits to Groningen were financed by a ZWO fellowship. Our work on gas in early-type galaxies is further supported by NATO Scientific Affairs Division through grant RG 098.82.

The Westerbork Radio Observatory is operated by the Netherlands Foundation for Radio Astronomy with financial support from ZWO. The observations were analyzed using GIPSY at the Computing Centre of Groningen University. We thank the Foundation staff and our local colleagues for their share in this work.

\section{REFERENCES}

Burstein, D. and Krumm, N.A. 1981, Astrophys. J. 250, 517

Dressler, A. and Sandage, A.R. 1982, Astrophys. J., in press Faber, S.M. and Gallagher, J.S. 1976, Astrophys. J. 204, 365

Larson, R.B., Tinsley, B.M., Caldwel1, C.N. 1980, Astrophys. J. 237, 692 Sancisi, R., van Woerden, H., Davies, R.D., Hart, L. 1982, in preparation Shane, W.W. 1980, Astron. Astrophys. 82, 314

Silk, J. and Norman, C.A. 1979, Astrophys. J. 234, 86 